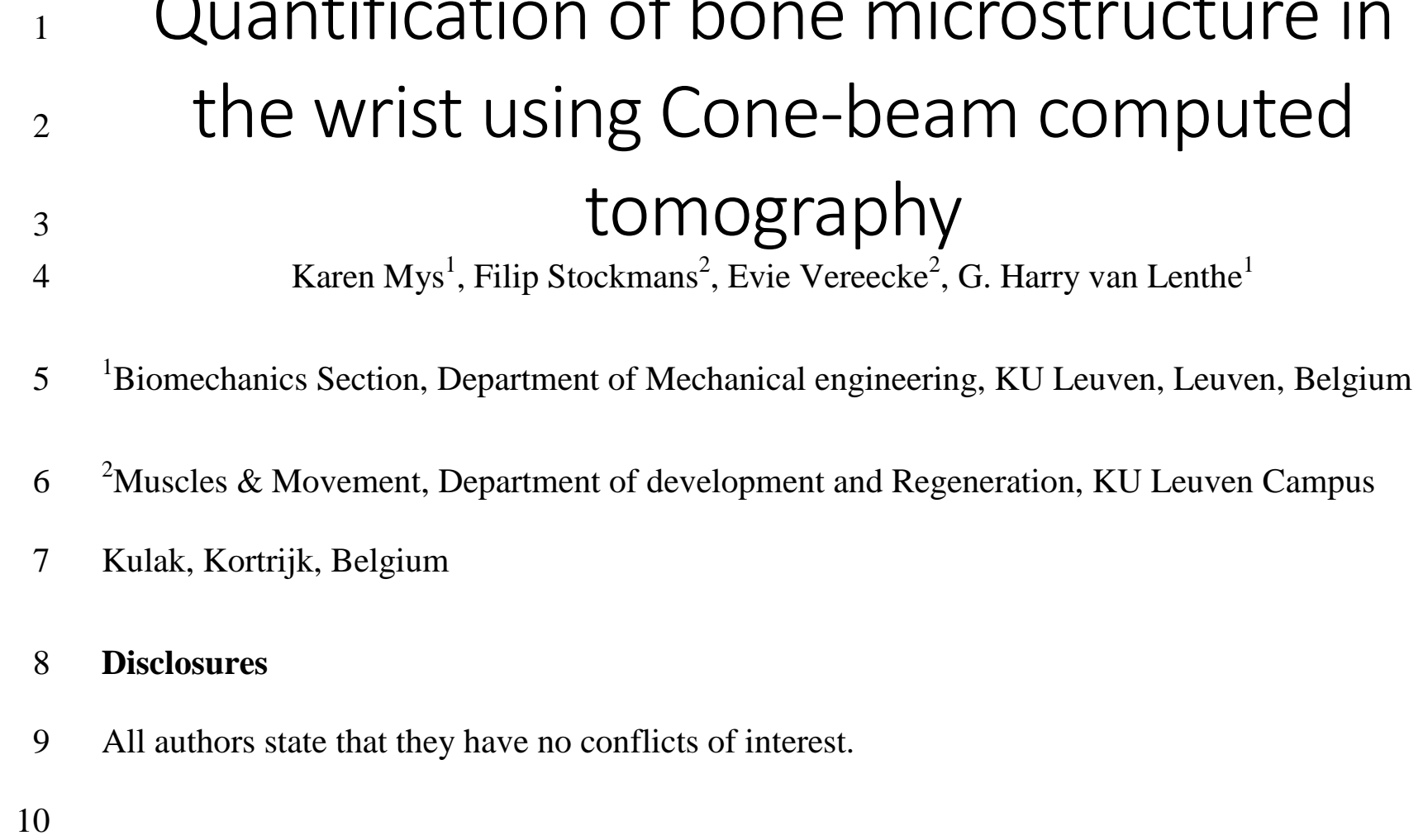

$5 \quad{ }^{1}$ Biomechanics Section, Department of Mechanical engineering, KU Leuven, Leuven, Belgium

$6 \quad{ }^{2}$ Muscles \& Movement, Department of development and Regeneration, KU Leuven Campus

$7 \quad$ Kulak, Kortrijk, Belgium

\section{Disclosures}

$9 \quad$ All authors state that they have no conflicts of interest.

\section{tomography}

Karen Mys ${ }^{1}$, Filip Stockmans ${ }^{2}$, Evie Vereecke ${ }^{2}$, G. Harry van Lenthe ${ }^{1}$ 


\section{Abstract}

12 Due to the rising life expectancy, bone diseases (e.g. osteoporosis, osteoarthritis) and trauma

13 (e.g. fracture) have become an important socio-economic burden. Accurate visualization and 14 quantification of the bone microstructure in vivo is seen as an important step to enhance 15 diagnosis and treatment. Micro-computed tomography (microCT) has become the gold standard 16 in three-dimensional (3D) imaging of trabecular bone structure. Yet, usage is limited to ex vivo 17 analyses, hence, it cannot be used to evaluate bone and bone adaptive responses in a patient. 18 High-resolution peripheral computed tomography (HR-pQCT) is considered the best technique 19 to measure the bone microarchitecture in vivo. By design HR-pQCT is limited to scanning 20 extremities, such as the distal radius and distal tibia with a limited field of view and long 21 scanning time ( 2 à $3 \mathrm{~min}$. for a stack of $0.9 \mathrm{~cm}$ ). Cone-beam computed tomography $(\mathrm{CBCT})$ is 22 a promising alternative with a much larger field of view. Yet, CBCT is challenged by artefacts 23 that reduce image contrast, such that it is currently being used for qualitative evaluation only. 24 Therefore, the aims of this work were first to enhance image contrast and second to determine 25 the accuracy of high-resolution CBCT for bone microarchitectural assessment.

26 Trapezia of nineteen female arthritic patients were scanned twice ex vivo; once using CBCT 27 (NewTom 5G, Cefla, Verona, Italy) at a nominal voxel size of $75 \mu \mathrm{m}$ and once using microCT 28 (SkyScan 1172, Bruker, Kontich, Belgium) at a voxel size of $19.84 \mu m$. The CBCT-scans were 29 reconstructed following 2 protocols: (1) using the commercial software delivered with the 30 scanner and (2) using in-house developed software. After reconstruction and image processing, 31 the images were segmented using adaptive thresholding. Bone morphometric parameters 32 including bone volume (BV), total tissue volume (TV), bone volume fraction (BV/TV), bone 33 surface density (BS/TV), trabecular thickness (Tb.Th), trabecular separation (Tb.Sp) and 
2

3

4

8

9

34 trabecular number (Tb.N) were calculated. Statistical evaluations were made at a significance

35 level of $5 \%$.

36 Significant correlations were found between the CBCT-based bone parameters and the microCT-

37 based parameters with $R^{2}>0.68$ The in-house reconstructed software outperformed the 38 commercial software. Better accuracy (overestimation of Tb.Th decreased from $114.24 \%$ to $3959.96 \%$ ) as well as higher correlations were observed for the in-house processed images. Still, a 40 significant overestimation was observed for BV/TV and Tb.Th and an underestimation for Tb.N.

41 We conclude that our CBCT image reconstruction improved image contrast which allowed for 42 an accurate quantification of trabecular bone microarchitecture.

\section{Keywords}

44 cone-beam computed tomography; bone parameters; micro-computed tomography; medical 45 imaging processing; quantification 


\subsection{Introduction}

47 Due to the aging population, bone diseases such as osteoporosis and osteoarthritis, are causing an 48 increased socio-economic burden [1]. Visualization and quantification of the bone microstructure 49 is often seen as an important step for a better understanding, diagnosis and follow up of bone 50 diseases [2]. Conventional medical imaging techniques, such as CT, X-ray and MRI, have 51 important shortcomings when applied to the visualization of bone micro-architecture. 52 Conventional CT and MRI can scan a large field of view, but its resolution (about $400 \mu \mathrm{m}$ ) is 53 inadequate to depict individual trabeculae (thickness $\sim 50-200 \mu \mathrm{m}$ ). X-ray imaging has a better 54 resolution, but its application towards visualization and quantification of bone micro-architecture 55 is limited due to the two-dimensional nature of the technique (projection images).

56 Several imaging techniques are currently being used to assess bone microarchitecture, of which 57 micro-computed tomography (microCT) has become the gold standard [3]. MicroCT provides 58 high-resolution, three-dimensional imaging of trabecular bone structure allowing comparison 59 between normal and diseased bone tissue. Yet, usage is limited to ex vivo analysis, hence, it 60 cannot be used to evaluate bone microstructure and adaptive responses in vivo. In vivo analysis 61 of bone structure at the distal forearm and ankle has recently become possible with the advent of 62 high-resolution peripheral quantitative CT (HR-pQCT) [4-7]. Two systems are currently on the 63 market (XtremeCT I + II, Scanco Medical, Brüttisellen, Switzerland), which provide a resolution 64 up to $60.7 \mu \mathrm{m}$. So far, this imaging technique has mainly been used in a research context and has 65 not found its way into daily clinical practice. One of the reasons for this is because scanning one 66 stack of $0.9 \mathrm{~cm}$ takes 2 to 3 min., making in vivo scanning of larger volumes of interest 67 challenging. 
68 A relatively new alternative is high-resolution Cone-beam computed tomography (CBCT) [8]. It

69 has become the gold standard in many dental and maxillofacial imaging applications due to its

70 high spatial resolution and low radiation dose (around $14 \mu \mathrm{Sv}$ for a FOV of 12x8 [9]) [10-12].

71 CBCT for musculoskeletal applications became possible with the advent of systems that looked

72 like a small $\mathrm{CT}$ and that could examine the patient in the supine position or systems with a ring

73 design through which an arm or leg could be put. The imaging of the fingers, wrist, elbow, foot,

74 ankle and knee joint using CBCT have been reported as well as imaging of long bones of the

75 extremities [13]. Despite the promising characteristics of high-resolution CBCT-scanners, i.e.

76 high spatial resolution $(75 \mu m)$, low radiation dose, fast acquisition, high versatility and cost-

77 effectiveness, the applicability of CBCT for musculoskeletal applications is still limited to 78 qualitative analyses, mainly due to imaging artefacts that reduce image contrast. Furthermore, 79 state-of-the-art CBCT-scanners are not calibrated to BMD. Therefore, the aims of this work were 80 (1) to enhance image contrast and (2) to determine the accuracy of high-resolution CBCT for 81 bone microarchitectural assessment.

\section{$83 \quad 1.2$ Materials and methods}

$84 \quad 1.2 .1$ Sample collection

85 A total of 19 trapezia (12 right, 7 left; 18 female, 1 male) were removed from the hands of 86 arthritic patients ranging in age from 53 to 76 years (mean 63.4 years) via trapeziectomy by a 87 certified hand surgeon (FS). The samples were stored directly after the operation at $-20^{\circ} \mathrm{C}$ and 88 were thawed prior to further analyses. 


\section{$90 \quad 1.2 .2$ Image acquisition}

91 Each sample was scanned ex vivo using Cone-beam computed tomography (CBCT, NewTom

$925 \mathrm{G}$, Cefla, Italy) at $75 \mu \mathrm{m}$, which is the best possible voxel size of this device. Next, the samples

93 were scanned using micro-computed tomography (microCT, SkyScan 1172, Bruker, Belgium) at

94 a voxel size of $19.84 \mu \mathrm{m}$. The samples were put in phosphate buffered saline during microCT

95 scanning to avoid dehydration (Table 1).

$96 \quad 1.2 .3 \quad$ Image processing

97 1.2.3.1 Image reconstruction

98 The processing and reconstruction of the projection data of the CBCT-images were performed 99 using the reconstruction software provided with the scanner as well as using in-house developed 100 reconstruction and image processing software (Fig. 1).

101 The in-house developed reconstruction started from the projection data. Firstly, filtering was 102 performed to reduce the detector noise present in the projection data. Afterwards the 103 polyenergetic ray sum, $p=-\ln \left(\frac{I_{D}}{I_{0}}\right)$, was calculated from the transmitted flux, $I_{D}$, and the blank 104 scan, $I_{0}$. The blank scan, $I_{0}$, was obtained by making a second scan without the bone, but with 105 the same scanner properties and the same bed position. The projection data was corrected for the 106 current modulation which was present during scanning. Afterwards, the images were 107 reconstructed at a slightly higher voxel size of $60 \mu \mathrm{m}$ by making use of an in-house developed 108 multithreaded FDK (Feldkamp, Davis and Kress) reconstruction program. A Ram-Lak high-pass 109 filter ([14]) was applied in the reconstruction program. In a second step, beam hardening 110 correction was performed using in-house developed software. No smoothing or other filtering 111 was done during the reconstruction or processing of the data. For the remainder of this paper, the 
112 terms RManufacturer, RInHouse, and RInHouseBeam and will be used to identify the images

113 reconstructed using the software that comes with the CBCT scanner, using our in-house

114 algorithm, and using our in-house algorithm with beam hardening correction, respectively.

\section{1.2.3.2 Image segmentation}

116 Segmentation of the CBCT-images was implemented in the program CTan (Bruker, Kontich, 117 Belgium) and is described in more detail in Appendix 1. The microCT-images were segmented 118 with a fixed global threshold and the noise was removed using a despeckler.

\section{$119 \quad$ 1.2.4 Image analysis}

120 For the microCT-images, the trabecular bone parameters were calculated in a trabecular volume 121 of interest (VOI) that was determined automatically following the method described by Buie et 122 al. [15]. The methodology, explained in more detail in Appendix 2, was adapted such that the 123 technique could also be used for bones without a well-defined cortex.

124 Using CTan ([16]) the following morphological parameters were calculated for the bone within 125 the trabecular mask in a direct way using a 3D analysis: bone volume fraction (BV/TV), 126 trabecular thickness (Tb.Th), trabecular separation (Tb.Sp) and trabecular number (Tb.N), bone 127 surface density (BS/TV). These analyses were performed for all image data, i.e., microCT, 128 RManufacturer, RInHouse, and RInHousebeam.

\section{$129 \quad 1.2 .5 \quad$ Statistical analysis}

130 Statistical analyses were performed in Matlab (The Mathworks, Natick, United States). To 131 compare microarchitectural parameters obtained with each imaging protocol, linear regression 132 was performed and the relative error $(\delta e)$ was calculated. For the statistical tests, the confidence 133 level $\alpha$ was set to 5\%. Finally, trendlines and Bland-Altman plots were generated for 
34 RInHouseBeam to assess the accuracy of CBCT in measuring the microstructural parameters. $135[17,18]$.

\section{$137 \quad 1.3$ Results}

138 Table 2 shows the coefficients of determination between the microstructural parameters as 139 obtained from MicroCT and those obtained from RManufacturer, RInHouse and 140 RInHouseBeam, respectively. Regression and Bland Altman plots between MicroCT and 141 RInHouseBeam are shown in Fig. 2.

142 Bone volume fraction (BV/TV), as determined using RInHouse and RInHouseBeam, was highly 143 correlated with the microCT analyses (Fig. 2a) though CBCT overestimated BV/TV. A much 144 higher correlation and accuracy was observed for RInHouseBeam $\left(R^{2}=0.69 ; \delta \epsilon=38.33 \%\right)$ 145 than for the two other protocols, RInHouse $\left(R^{2}=0.65 ; \delta \epsilon=48.20 \%\right)$ and RManufacturer $146 \quad\left(R^{2}=0.60 ; \delta \epsilon=55.14 \%\right)$.

147 All CBCT protocols significantly overestimated trabecular thickness (Fig. 2b). The in-house 148 reconstruction protocols (RInHouse and RInHouseBeam) overestimated the trabecular thickness 149 (Tb.Th) with an average of $108 \mu m(\delta e=59.96 \%)$ and $120 \mu m(\delta e=66.39 \%)$, 150 respectively, whereas the average overestimation was $206 \mu m(\delta e=114.24 \%)$ when the 151 scanner's reconstruction (RManufacturer) was used.

152 Trabecular separation (Tb.Sp) was highly correlated with the results obtained via microCT, and 153 this for all the reconstructions (Fig. 2c). Tb.Sp was slightly underestimated $(\delta e=-4.45 \%)$ by 154 RInHouse and slightly overestimated by RInHouseBeam ( $\delta e=0.98 \%)$, respectively. The 

$71569.43 \%)$.

155 reconstructed images by the scanners software (RManufacturer) overestimated $\mathrm{Tb} . \mathrm{Sp}(\delta e=$

157 Trabecular number (Tb.N) (Fig. 2d) calculated from CBCT images was highly correlated with 158 the data obtained from microCT, for all reconstructions. A higher bias was observed for 159 RManufacturer $(\delta e=-27.74 \%)$ than for the in-house reconstruction $(\delta e=-10.91 \%$ for 160 RInHouse and $\delta e=-13.53 \%$ for RInhouseBeam).

161 A high correlation was observed between bone surface (BS/TV) (Fig. 2e) obtained from the $162 \mathrm{CBCT}$ - and microCT-images. RInHouse $(\delta e=-9.7 \%)$ and RInHouseBeam $(\delta e=$ $163-10.45 \%)$ and slightly underestimated the BS/TV. The bias was substantially higher $(\delta e=$ $164-28.99 \%)$ when the reconstruction by the manufacturer was used.

\subsection{Discussion}

The aims of this study were (1) to enhance image contrast and (2) to determine the accuracy of high-resolution CBCT for bone microarchitectural assessment. Both aims have been achieved. For the first aim, we implemented a multithreaded $\mathrm{C}++$ code to process the projection data, to reconstruct a 3D-image and to perform image correction afterwards. For the second aim, we 174 study.

175 We demonstrated that the in-house processed images (RInHouse and RInHouseBeam) 176 outperformed the reconstructed images using the scanner's reconstruction software 
177 (RManufacturer). Higher correlations and better accuracy (systematic smaller over- or 178 underestimation) were obtained. The beam hardening correction applied in RInHouseBeam 179 improved the accuracy significantly against RInHouse. Tb.Sp was slightly underestimated $180(\delta e=-4.45 \%)$ by RInHouse and slightly overestimated by RInHouseBeam ( $\delta e=0.98 \%)$, 181 respectively. This is in accordance with an overestimation of Tb.Th. In contrast to the in-house 182 processed images, RManufacturer overestimated Tb.Sp significantly $(\delta e=9.43 \%)$. This can be 183 explained by the large number of thin trabeculae that were not detected in RManufacturer as well 184 as by the presence of spatially closely related trabeculae that were depicted as one thick 185 trabeculae instead.

186 We also demonstrated that $\mathrm{CBCT}$ has a high potential to visualize and quantify the bone 187 microstructure for musculoskeletal applications. Significant correlations between the CBCT188 images and the microCT-images were observed for all bone parameters (BV/TV, BS/TV, Tb.Th, 189 Tb.Sp and Tb.N).

190 An additional strength of this study was that the segmentation of all the images was done 191 following automated protocols in a completely automatic way that did not require any user 192 interaction. Not only did this eliminate the subjective impact of interpretation, it also eliminated 193 the tedious and time-consuming masking procedure.

194 This is the first study to report on the quantification of bone microstructure using CBCT for 195 musculoskeletal applications. A few studies have presented CBCT-based bone microstructure for 196 dental applications $[12,19,20]$. In the analyses for dental purposes, the overestimation in Tb.Th 197 was at least $184 \%$ [19] and $112 \%$ [20]. The latter corresponds quite well to the overestimation 198 found for RManufacturer (114.24\%) in our study. In the present study, we found an 199 overestimation of $59.96 \%$ when using the RInHouseBeam protocol which represents an 
important improvement compared to the previous studies. Furthermore, the correlations obtained in the present study are among the highest published in literature $[19,20]$. In addition, it should 202 be noted that in the present study fresh-frozen trapezia of severely osteoarthritic patients were 203 used whereas dry defatted bones were used in the dental applications. We hypothesize that the 204 segmentation of defatted bone is easier than the bones used here because the image contrast 205 between bone and soft tissue is smaller than the contrast between bone and air. The use of 206 severely osteoarthritic bones with large variations in trabecular thickness is likely to have 207 complicated the segmentation; hence, we hypothesize that the analyses of the trapezia represents a more challenging case than those of the bones for dental applications. Yet, considering the use of samples from other anatomical locations and considering that in the present study we used severely osteoarthritic bone, limits a direct comparison to previous studies.

211 An alternative to using $\mathrm{CBCT}$ in the visualization and quantification of bone microstructure is 212 using high-resolution peripheral quantitative computed tomography (HR-pQCT). The 213 correlations achieved in this study were higher than those obtained using the first generation HR214 pQCT (XTremeCT, voxel size 82 $\mu \mathrm{m}$ ) [21] (analysed using direct voxel-based measurements of 215 structure), but inferior to the second generation HR-pQCT (XTremeCT-II, voxel size $61 \mu \mathrm{m}$ ) 216 [22]. Yet, because the HR-pQCT studies have evaluated other anatomical sites than we evaluated 217 using CBCT, a direct comparison cannot be made. In future work, we will extend our comparison to HR-pQCT.

219 Three trapezia contained a microstructure that was characterized by many thin trabeculae, 220 defined as containing more than $40 \%$ of the trabeculae with a thickness below $120 \mu \mathrm{m}$, which represents less than 2 voxels in the in-house reconstructed CBCT-images. Those bones were 222 marked in Fig. 2 and all analyses were repeated with those three samples excluded (Table 3). 
Those images appeared blurry and could not be segmented accurately. Indeed, the coefficients of determinations were in general higher when those bones were excluded. An exception was Tb.Th; due to the exclusion of the three samples with the lowest trabecular thickness, the spread in values became smaller leading to a lower coefficient of determination. Relevant for clinical application is that these trapezia can be detected automatically using the in-house reconstructed CBCT-images; specifically, in RInHouseBeam, these samples have more than $19 \%$ of trabeculae with a thickness below $180 \mu m$. Hence, for carpal bones with a high ratio of thin trabeculae, the direct method for calculating the parameters should be avoided in clinical practice. We hypothesize that the limit of $120 \mu \mathrm{m}$ will be lower when scanning radii because of these contain more regular trabeculae. Further studies will be performed to test this.

Because of the use of severe osteoarthritic bones, some samples contained trabeculae with small holes inside, nearly parallel trabeculae and very irregular bone structures (Fig 3) which were undetectable by CBCT. Quantitative analyses showed that the spacings and cavities were often smaller than $60 \mu m$; hence, whereas those trabeculae were treated as two small trabeculae in the microCT-analyses, whereas they were treated as one thick trabecula in CBCT analyses. Especially, three bones (indicated with a cross in Fig. 2) contained a lot of those features and influenced the correlations of Tb.Th and BV/TV significantly. When those bones would not be present in this study, the correlation for Tb.Th would increase from $R^{2}=0.50$ to $R^{2}=0.84$ 241 and for $\mathrm{BV} / \mathrm{TV}$ from $R^{2}=0.75$ to $R^{2}=0.92$.

242 It is often claimed that scattering limits the quality of high-resolution CBCT-images [11]. We 243 hypothesize that for small bones such as the trapezium, the partial volume effect and beam hardening effect present the biggest artefacts on the CBCT-images. Indeed, in this study we 
showed that beam hardening correction enhanced the images tremendously. The partial volume effect was not corrected for.

247 A potential limitation towards clinical application is that in the present study the bones were scanned in isolation and not in their anatomical configuration. Hence, external artefacts that occur during in vivo scanning were not present. Artefacts due to motion and artefacts due to beam hardening by surrounding bones could potentially deteriorate image quality; in addition, scattering, which is known to increase with increasing object volume, could cause artefacts. These effects will be addressed in subsequent studies.

\subsection{Conclusion}

We conclude that bone microarchitecture of fresh-frozen arthritic trapezia of severely osteoarthritic patients can be measured with good accuracy from CBCT-images. We also showed that image quality can be significantly enhanced in comparison with state-of-the-art software, without a substantial increase in compute time. Further artefact identification and corrections seems useful as well as comparison with HR-pQCT.

We hypothesize that CBCT imaging and analyses could find application in patients with bone diseases such as osteoporosis and osteoarthritis, in the detection of occult fractures which are invisible or doubtful on conventional X-rays, for the visualization of severe fractures to aid surgical intervention and for the follow up of those interventions/fracture repair.

\section{Acknowledgments:}


The project was funded in part by the KU Leuven Internal Funds (C24/16/027). The authors would like to thank Dr. Olivier Vanovermeire for assistance with the CBCT-scans and Haniyeh Hemmatian, $\mathrm{PhD}$, for assistance with the microCT-scans. The authors would like to thank Dr. Walter Coudyzer for providing access to the European Spine Phantom (ESP) and the European Forearm Phantom (EFP) that were used in the initial stage of the study.

\section{References}

[1] E. Hernlund, A. Svedbom, M. Ivergård, J. Compston, C. Cooper, J. Stenmark, E. V. McCloskey, B. Jönsson, J.A. Kanis, Osteoporosis in the European Union: Medical management, epidemiology and economic burden: A report prepared in collaboration with the International Osteoporosis Foundation (IOF) and the European Federation of Pharmaceutical Industry Associations (EFPIA), Arch. Osteoporos. 8 (2013). doi:10.1007/s11657-013-0136-1.

[2] X.S. Liu, X.H. Zhang, K.K. Sekhon, M.F. Adam, D.J. McMahon, J.P. Bilezikian, E. Shane, X.E. Guo, High-Resolution Peripheral Quantitative Computed Tomography Can Assess Microstructural and Mechanical Properties of Human Distal Tibial Bone, J. Bone Miner. Res. 25 (2009) 746-756. doi:10.1359/jbmr.090822.

[3] R. Müller, H. Van Campenhout, B. Van Damme, G. Van Der Perre, J. Dequeker, T. Hildebrand, P. Rüegsegger, Morphometric Analysis of Human Bone Biopsies: A Quantitative Structural Comparison of Histological Sections and Micro-Computed Tomography, Bone. 23 (1998) 59-66. doi:10.1016/S8756-3282(98)00068-4.

[4] S. Boutroy, M.L. Bouxsein, F. Munoz, P.D. Delmas, In vivo assessment of trabecular bone microarchitecture by high-resolution peripheral quantitative computed tomography, 
J. Clin. Endocrinol. Metab. 90 (2005) 6508-6515. doi:10.1210/jc.2005-1258.

[5] S. Boutroy, B. Van Rietbergen, E. Sornay-Rendu, F. Munoz, M.L. Bouxsein, P.D. Delmas, Finite element analysis based on in vivo HR-pQCT images of the distal radius is associated with wrist fracture in postmenopausal women, J. Bone Miner. Res. 23 (2008) 392-399. doi:10.1359/jbmr.071108.

[6] N. Vilayphiou, S. Boutruy, E. Sornay-Rendu, B. van Rietbergen, F. Munoz, P.D. Delmas, R. Chapurlat, Finite element analysis performed on radius and tibia HR-pQCT images and fragility fractures at all sites in postmenopausal women, Bone. 46 (2010) 1030-1037.

[7] K. Engelke, J.E. Adams, G. Armbrecht, P. Augat, C.E. Bogado, M.L. Bouxsein, D. Felsenberg, M. Ito, S. Prevrhal, D.B. Hans, E.M. Lewiecki, Clinical use of quantitative computed tomography and peripheral quantitative computed tomography in the management of osteoporosis in adults: The 2007 ISCD official positions, J. Clin. Densitom. Assess. Skelet. Heal. 11 (2008) 123-162. doi:10.1016/j.jocd.2007.12.010.

[8] W.C. Scarfe, a G. Farman, P. Sukovic, Clinical applications of cone-beam computed tomography in dental practice, J Can Dent Assoc. 72 (2006) 75-80. doi:10.1016/j.tripleo.2005.07.027.

[9] J. Koivisto, T. Kiljunen, N. Kadesjö, X.Q. Shi, J. Wolff, Effective radiation dose of a MSCT, two CBCT and one conventional radiography device in the ankle region, J. Foot Ankle Res. 8 (2015). doi:10.1186/s13047-015-0067-8.

[10] J. De Cock, K. Mermuys, J. Goubau, S. Van Petegem, B. Houthoofd, J.W. Casselman, Cone-beam computed tomography: A new low dose, high resolution imaging technique of the wrist, presentation of three cases with technique, Skeletal Radiol. 41 (2012) 93-96. 
doi:10.1007/s00256-011-1198-z.

[11] Y. Huang, J. Van Dessel, M. Depypere, M. Ezeldeen, A.A. Iliescu, E. Dos, Validating cone-beam computed tomography for peri-implant bone morphometric analysis, Bone Res. 2 (2014). doi:10.1038/boneres.2014.10.

314 [12] N. Ibrahim, A. Parsa, B. Hassan, P. van der Stelt, I.H. a Aartman, D. Wismeijer, Accuracy of trabecular bone microstructural measurement at planned dental implant sites using cone-beam CT datasets, Clin. Oral Implants Res. 25 (2014) 941-945. doi:10.1111/clr.12163.

[13] W. Zbijewski, P. De Jean, P. Prakash, Y. Ding, J.W. Stayman, N. Packard, R. Senn, D. Yang, J. Yorkston, A. Machado, J.A. Carrino, J.H. Siewerdsen, A dedicated cone-beam CT system for musculoskeletal extremities imaging: Design, optimization, and initial performance characterization, Med. Phys. 38 (2011) 4700-4713. doi:10.1118/1.3611039.

322 [14] S. Lee, C. Lee, H. Cho, H. Park, D. Kim, Y. Choi, H. Kim, L. Seung-Wan, L. Chang-Lae, C. Hyo-Min, P. Hye-Suk, K. Dae-Hong, C. Yu-Na, K. Hee-Joung, Effects of Reconstruction Parameters on Image Noise and Spatial Resolution in Cone-beam Computed Tomography, J. Korean Phys. Soc. 59 (2011) 2825-2832. doi: $10.3938 / \mathrm{jkps} .59 .2825$.

[15] H.R. Buie, G.M. Campbell, R.J. Klinck, J. a. MacNeil, S.K. Boyd, Automatic segmentation of cortical and trabecular compartments based on a dual threshold technique for in vivo micro-CT bone analysis, Bone. 41 (2007) 505-515. doi:10.1016/j.bone.2007.07.007.

[16] Bruker, No Title, (n.d.). 
[17] J.M. Bland, D.G. Altman, Measuring agreement in method comparison studies., Stat. Methods Med. Res. 8 (1999) 135-160. doi:10.1191/096228099673819272.

[18] J. Martin Bland, D. Altman, Statistical methods for assessing agreement between two methods of clinical measurement, Lancet. 327 (1986) 307-310. doi:10.1016/S01406736(86)90837-8.

[19] E. Klintström, Smedby, B. Klintström, T.B. Brismar, R. Moreno, Trabecular bone histomorphometric measurements and contrast-to-noise ratio in CBCT, Dentomaxillofacial Radiol. 43 (2014). doi:10.1259/dmfr.20140196.

[20] J. Van Dessel, L. Ferreira, P. Nicolielo, Y. Huang, P. Slagmolen, C. Politis, I. Lambrichts, R. Jacobs, N. Giano, N. Generation, N. Giano, Quantification of bone quality using different cone beam computed tomography devices : Accuracy assessment for edentulous human mandibles, 9 (2016) 411-424.

[21] J. a MacNeil, S.K. Boyd, Accuracy of high-resolution peripheral quantitative computed tomography for measurement of bone quality., Med. Eng. Phys. 29 (2007) 1096-1105. doi:10.1016/j.medengphy.2006.11.002.

[22] S.L. Manske, Y. Zhu, C. Sandino, S.K. Boyd, Human trabecular bone microarchitecture can be assessed independently of density with second generation HR-pQCT, Bone. 79 (2015) 213-221. doi:10.1016/j.bone.2015.06.006.

[23] D.P. Shamonin, E.E. Bron, B.P.F. Lelieveldt, M. Smits, S. Klein, M. Staring, Fast parallel image registration on CPU and GPU for diagnostic classification of Alzheimer's disease., Front. Neuroinform. 7 (2014). doi:10.3389/fninf.2013.00050. 
[24] S. Klein, M. Staring, K. Murphy, M.A. Viergever, J.P.W. Pluim, elastix: A Toolbox for Intensity-Based Medical Image Registration, IEEE Trans. Med. Imaging. 29 (2010) 196205. doi:10.1109/TMI.2009.2035616.

\subsection{Appendix 1: segmentation of CBCT-images}

358 The segmentation of the CBCT-images was performed automatically (Fig. A1). First, a global 359 pre-threshold was performed to delineate a volume of interest (VOI) in which adaptive 360 thresholding was applied. The global pre-threshold was chosen in such a way that all the 361 trabeculae were included. For every voxel in the VOI an adaptive threshold was calculated as the 362 mean of the minimal and maximal values in a sphere with a radius of 6 pixels around the voxel. 363 In parallel, a high global threshold was applied as to include the bone with relatively low grey 364 values that would go unselected by the adaptive segmentation process. Finally, both 365 segmentations were added together and a despeckle of 5 pixels was used to remove the noise.

\subsection{Appendix 2: automatic calculation of volume of interest based on the microCT-} images and registration of the mask on the other images

369 A trabecular volume of interest (VOI) was selected automatically (Fig. A2) based on the 370 microCT-images, following the method described by Buie et al. [15]. The methodology was 371 adapted (indicated in red in Fig. A3) such that the technique could also be used for bones without 372 a well-defined cortex. In the work of Buie et al. the trabecular mask was determined in a two373 step procedure by subtracting the segmented image from the total mask of the bone, followed by 374 a closing operation. We have replaced the first step with two different operations. First, the 
segmented image was dilated with 3 pixels in order to create a more continuous cortical shell. Afterwards, the dilated segmented image was subtracted from the total mask of the bone.

377 Second, we assumed the thickness of the cortical shell to be at least 6 pixels. These assumptions 378 assured that the trabecular mask would not penetrate into the pores of the cortex. It was 379 implemented by making use of a 6 pixel erosion of the whole mask followed by extraction of this 380 mask.

$3813 \mathrm{D}$ intensity-based image registration of the gray value-images was used to select identical 382 trabecular volumes in the CBCT-images. The registration consisted out of four automatic steps 383 and was performed after the correction for beam hardening (RInHouseBeam). First, masks of the 384 whole bones were automatically determined (mask all bone in Fig. A3). Those masks were used 385 to get a rough, but fast, registration of the images. Specifically, the translation between the two 386 masks was calculated based on the center of gravity and the rotation was based on the axes of 387 inertia. Second, the masks were registered using the open-source software Elastix [23,24]. Third, 388 Elastix was used to perform a precise registration between the grey-level images RInHouseBeam 389 and microCT. Fourth, the inverse transformation was applied to transform the microCT-based 390 trabecular mask onto the CBCT-images. 


\section{Tables}

393 Table 1: Scanning parameters of the CBCT NewTom 5G (Cefla, Verona, Italy) and microCT

394 Skyscan-1172 (Bruker, Kontich, Belgium). [1-column fitting table]

Table 2: Relative error $(\delta \mathrm{e})$ with standard deviation and coefficients of determination between microCT and RManufacturer (standard scanner's reconstruction), RInHouse (in house reconstruction) and RInHouseBeam (in house reconstruction with beam hardening correction) for all samples $(\mathrm{N}=19)$. RInHouseBeam outperformed RManufacturer and provided better accuracy as well as higher correlations. [2-column fitting table]

Table 3: Relative error $(\delta \mathrm{e})$ with standard deviation and coefficients of determination between microCT and RManufacturer (standard scanner's reconstruction), RInHouse (in house reconstruction) and RInHouseBeam (in house reconstruction with beam hardening correction). Three bone samples with very low trabecular thickness were excluded $(\mathrm{N}=16)$. [2-column fitting table] 


\section{Figures}

410 Figure 1: The reconstructed images (a, c, e, g) and the corresponding segmented images (b, d, f, 411 h). (a, b) microCT-image; (c,d) CBCT-image reconstructed using the standard scanner software 412 (RManufacturer); (e, f) in-house reconstructed image (RInHouse); $(\mathrm{g}, \mathrm{h})$ in-house reconstructed 413 image with beam-hardening correction. [2-column fitting image - MicroCTBigRaw, 414 MicroCTBigSegmented, CeflaRaw, CeflaSegmented, CBCTInHouseRaw, 415 CBCTInHouseSegmented, CBCTInHouseBeamRaw and CBCTInHouseBeamSegmented]

417 Figure 2: Regression plots and Bland Altman plots between MicroCT and RInHouseBeam for 418 bone volume fraction (BV/TV), trabecular thickness (Tb.Th), trabecular separation (Tb.Sp), 419 trabecular number (Tb.N), and bone surface density (BS/TV). Three samples with low trabecular 420 thickness (more than $40 \%$ of the trabeculae in the VOI thinner than $120 \mu \mathrm{m}$ ) are indicated with 421 filled dots. Three samples that contained a lot of detailed architectural features (almost parallel 422 trabeculae close to each other, small holes and thin, irregular trabeculae) are indicated with a 423 cross. [2-column fitting image - BVTVVersion2, TbThVersion2, TbSpVersion2, TbNVersion2 424 and BSVersion2]

426 Figure 3: Small bone features such as small holes in the trabeculae (a), nearly parallel trabeculae 427 close to each other (b) and thin, irregular trabecular structures (c) were observed in the microCT428 images (top). The resolution of the CBCT-images (bottom) is too low to depict these features, thus causing morphometric differences between $\mathrm{CBCT}$ and microCT. [2-column fitting image - 
SmalHolesMicroCT, NearlyParallelTrabMicroCT, ThinIrregularTrabeculaeMicroCT,

SmalHolesCBCT, NearlyParallelTrabCBCT and ThinIrregularTrabeculaeCBCT]

Figure A1 (Figure 4): Automatic segmentation technique for the CBCT-images. The main part 434 consists of a low global threshold, followed by an adaptive segmentation in a sphere with a 435 radius of 6 pixels. In parallel, a high global threshold was applied as to include the bone with 436 relatively low grey values that would go unselected by the adaptive segmentation process. 437 Finally, the noise is reduced with a despeckler. [2-column fitting image 438 AdaptiveSegmentation2]

440 Figure A2 (Figure 5): Depicted in red is one section through the volume of interest (VOI) which 441 was used for the microstructural analyses. This VOI is determined automatically from the 442 microCT-images. [1-column fitting image, color - VOI]

444 Figure A3 (Figure 6): Automatic determination of the volume of interest (VOI). Based on the 445 method by Buie et al [14] several steps were added (marked with red labels) to adapt it for the 446 analyses of CBCT-images. [2-column fitting image, full page, color - VOIKM] 


\title{
Quantification of bone microstructure in the wrist using Cone-beam computed tomography: tables
}

\author{
Karen Mys, Filip Stockmans, Evie Vereecke, G.Harry van Lenthe
}

KU Leuven

\begin{tabular}{l|cc} 
& CBCT NewTom 5G & MicroCT Skyscan \\
\hline Energy $(\mathrm{kVp})$ & 110 & 100 \\
Current $(\mu A)$ & $7-13$ & 100 \\
FOV $(\mathrm{cm} \mathrm{cm} \times \mathrm{cm})$ & $6 \times 6 \times 6$ & $2 \times 2 \times 1$ \\
Projections & 486 & 512 \\
Isotropic voxel size $(\mu \mathrm{m})$ & 75 & 19.84
\end{tabular}

Table 1: Scanning parameters of the CBCT NewTom 5G (Cefla, Verona, Italy) and microCT Skyscan 1172 (Bruker, Kontich, Belgium). [1-column fitting table]

\begin{tabular}{|c|c|c|c|c|c|c|c|}
\hline & & \multicolumn{6}{|c|}{$\operatorname{CBCT}(\mathrm{N}=19)$} \\
\hline & \multirow{2}{*}{$\begin{array}{r}\text { MicroCT } \\
\text { Mean(SD) }\end{array}$} & \multicolumn{2}{|c|}{ RManufacturer } & \multicolumn{2}{|c|}{ RInHouse } & \multicolumn{2}{|c|}{ RInHouseBeam } \\
\hline & & $\delta e(S D)$ & $R^{2}$ & $\delta e(S D)$ & $R^{2}$ & $\delta e(S D)$ & $R^{2}$ \\
\hline $\mathrm{BV} / \mathrm{TV}[\%]$ & $23.38(2.8)$ & $55.14(11.5) \%$ & 0.60 & $48.20(10.4) \%$ & 0.65 & $38.33(9.9) \%$ & 0.69 \\
\hline Tb.Th $[m m]$ & $0.18(0.02)$ & $114.24(17.9) \%$ & 0.52 & $66.39(11.0) \%$ & 0.68 & $59.96(10.2) \%$ & 0.68 \\
\hline Tb.Sp $[\mathrm{mm}]$ & $0.61(0.07)$ & $9.43(5.7) \%$ & 0.84 & $-4.45(5.4) \%$ & 0.81 & $0.98(7.0) \%$ & 0.73 \\
\hline Tb.N $[1 / m m]$ & $1.30(0.11)$ & $-27.74(3.4) \%$ & 0.63 & $-10.91(3.3) \%$ & 0.82 & $-13.53(4.0) \%$ & 0.81 \\
\hline $\mathrm{BS} / \mathrm{TV}[1 / \mathrm{mm}]$ & $4.79(0.35)$ & $-28.99(3.5) \%$ & 0.50 & $-9.7(4.0) \%$ & 0.72 & $-10.45(4.5) \%$ & 0.72 \\
\hline
\end{tabular}

Table 2: Relative error $(\delta \epsilon)$ with standard deviation and coefficients of determination between microCT and RManufacturer (standard scanner's reconstruction), RInHouse (in house reconstruction) and RInHouseBeam (in house reconstruction with beam hardening correction) for all samples $(N=19)$. RInHouseBeam outperformed RManufacturer and provided better accuracy as well as higher correlations. [2-column fitting table]

\begin{tabular}{|c|c|c|c|c|c|c|c|}
\hline & \multirow{3}{*}{$\begin{array}{r}\text { MicroCT } \\
\text { Mean(SD) }\end{array}$} & \multicolumn{6}{|c|}{$\operatorname{CBCT}(\mathrm{N}=16)$} \\
\hline & & \multicolumn{2}{|c|}{ RManufacturer } & \multicolumn{2}{|c|}{ RInHouse } & \multicolumn{2}{|c|}{ RInHouseBeam } \\
\hline & & $\delta e(S D)$ & $R^{2}$ & $\delta e(S D)$ & $R^{2}$ & $\delta e(S D)$ & $R^{2}$ \\
\hline $\mathrm{BV} / \mathrm{TV}[\%]$ & $23.98(2.45)$ & $53.11(10.4) \%$ & 0.54 & $46.29(8.6) \%$ & 0.68 & $37.14(6.9) \%$ & 0.75 \\
\hline Tb.Th $[\mathrm{mm}]$ & $0.19(0.01)$ & $109.36(12.0) \%$ & 0.53 & $64.06(9.5) \%$ & 0.54 & $58.50(10.0) \%$ & 0.50 \\
\hline Tb.Sp $[\mathrm{mm}]$ & $0.62(0.07)$ & $8.63(4.7) \%$ & 0.85 & $-5.24(4.3) \%$ & 0.86 & $-0.07(4.9) \%$ & 0.83 \\
\hline Tb.N $[1 / m m]$ & $1.29(0.10)$ & $-26.89(2.5) \%$ & 0.80 & $-10.67(2.8) \%$ & 0.83 & $-13.34(2.6) \%$ & 0.86 \\
\hline $\mathrm{BS} / \mathrm{TV}[1 / \mathrm{mm}]$ & $4.75(0.31)$ & $-28.23(2.8) \%$ & 0.62 & $-10.24(4.0) \%$ & 0.66 & $-10.49(3.8) \%$ & 0.69 \\
\hline
\end{tabular}

Table 3: Relative error ( $\delta$ e) with standard deviation and coefficients of determination between microCT and RManufacturer (standard scanner's reconstruction), RInHouse (in house reconstruction) and RInHouseBeam (in house reconstruction with beam hardening correction). Three bone samples with very low trabecular thickness were excluded $(N=16)$. [2-column fitting table] 


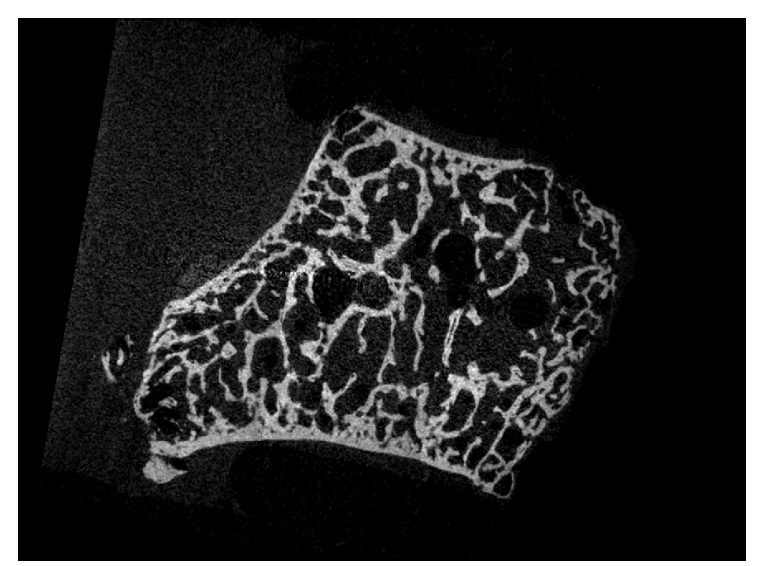

(a) $\operatorname{MicroCT}(19.84 \mu m)$

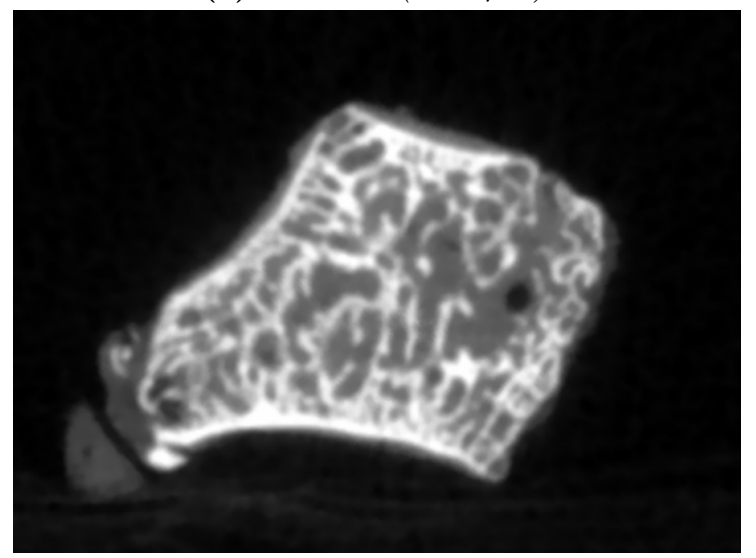

(c) CBCT RManufacturer $(75 \mu \mathrm{m})$

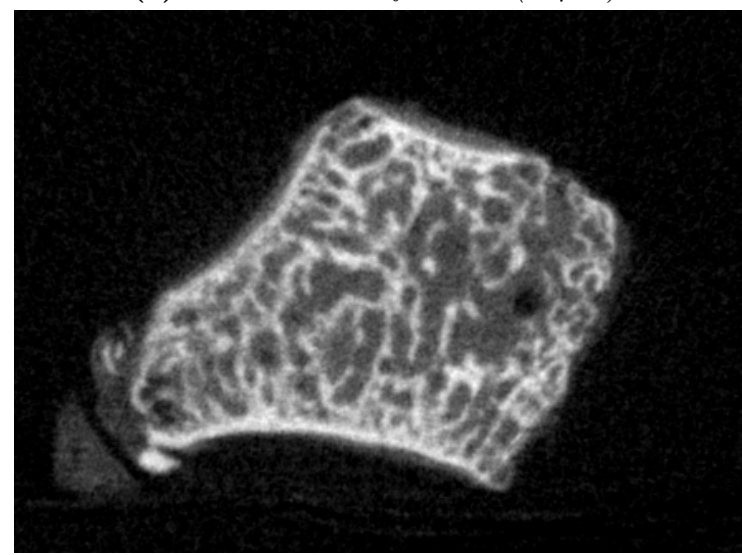

(e) $C B C T$ RInHouse $(60 \mu m)$

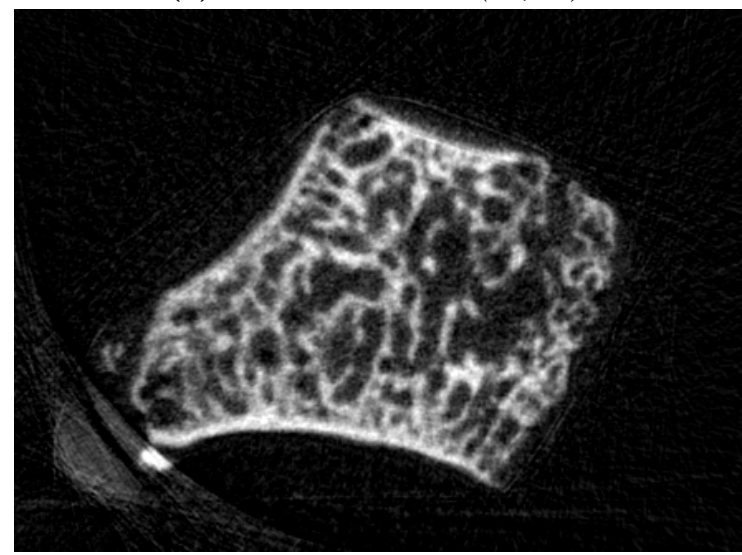

(g) CBCT RInHouseBeam $(60 \mu m)$

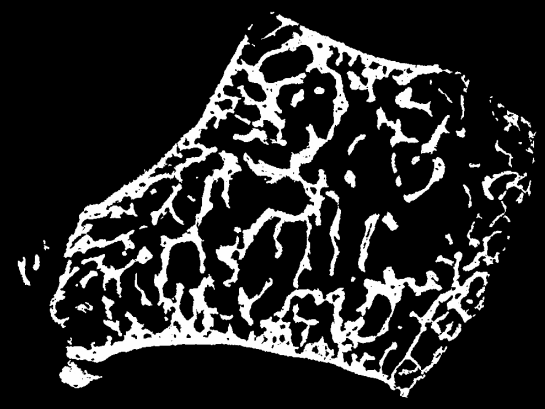

(b) MicroCT segmented

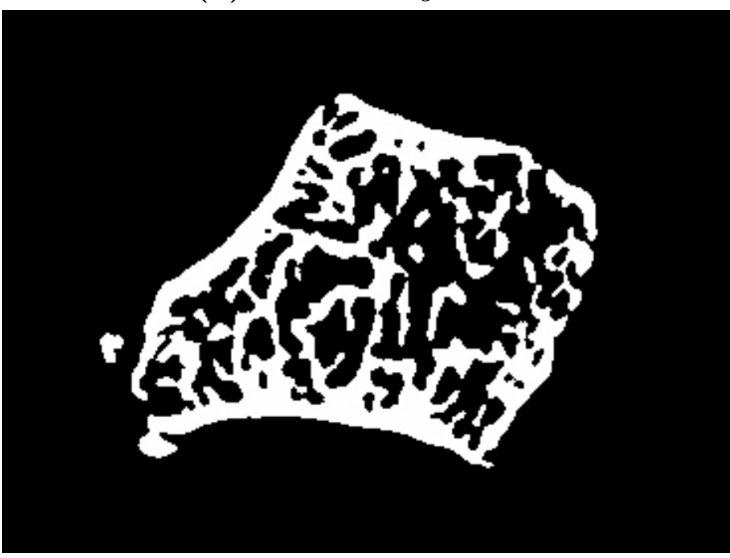

(d) CBCT RManufacturer segmented

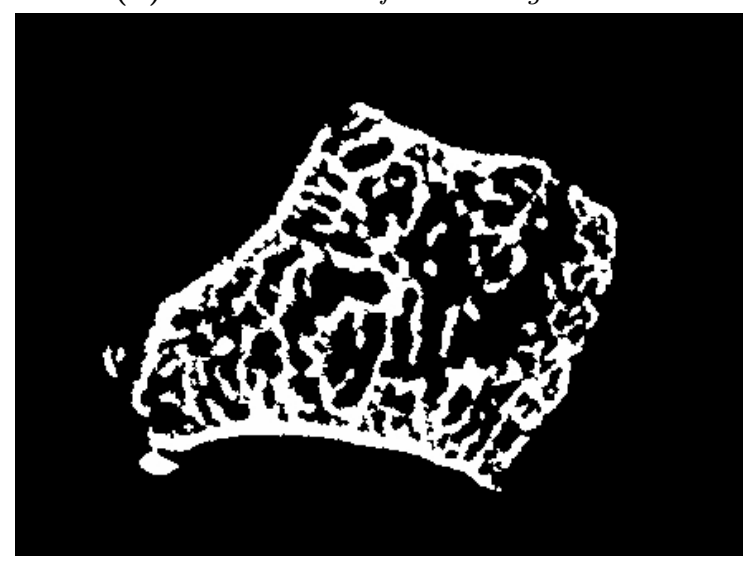

(f) CBCT RInHouse segmented

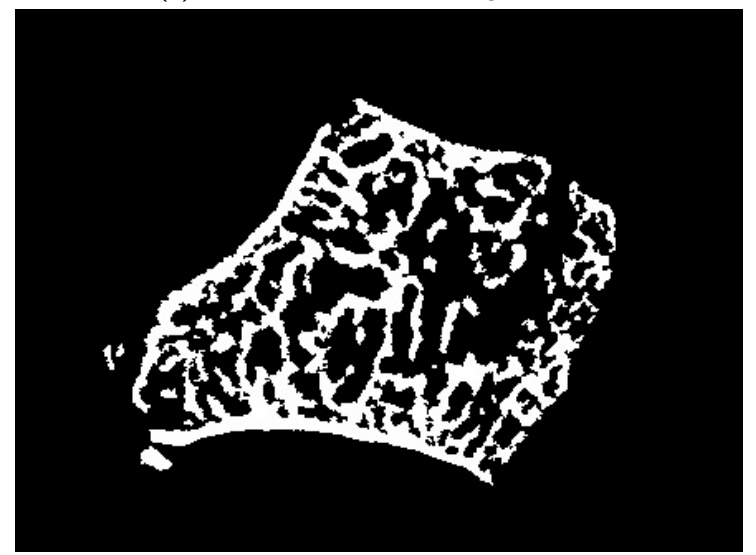

(h) CBCT RInHouseBeam segmented

Figure 1: The reconstructed images $(a, c, e, g)$ and the corresponding segmented images (b, $d, f, h)$. ( $a, b)$ microCTimage; (c,d) CBCT-image reconstructed using the standard scanner software (RManufacturer); (e, f) in-house reconstructed image (RInHouse); $(g, h)$ in-house reconstructed image with beam-hardening correction. [2-column fitting image] 

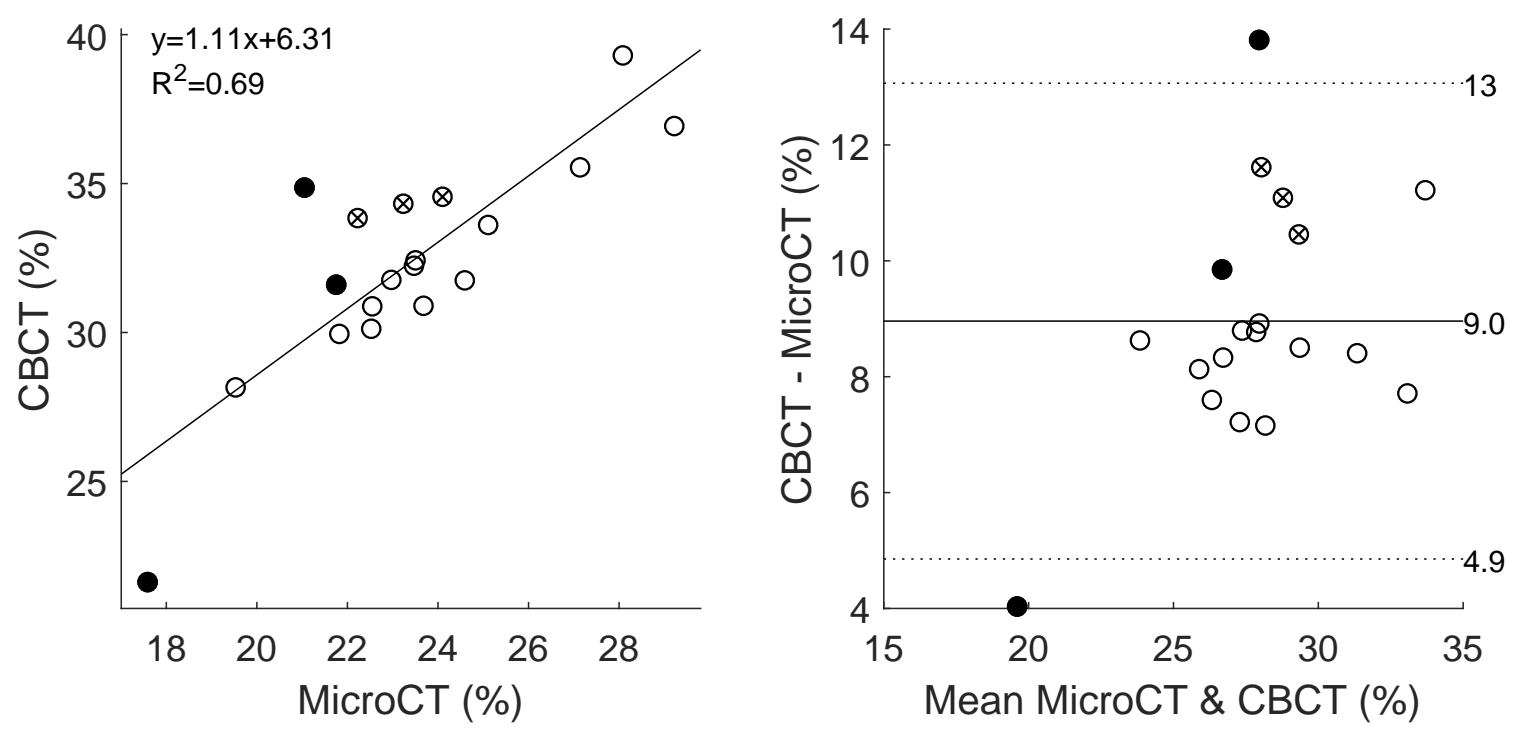

(a) Percent bone volume $(B V / T V)$

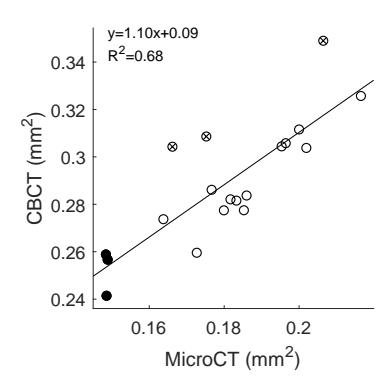

(b) Trabecular thickness (Tb.Th)
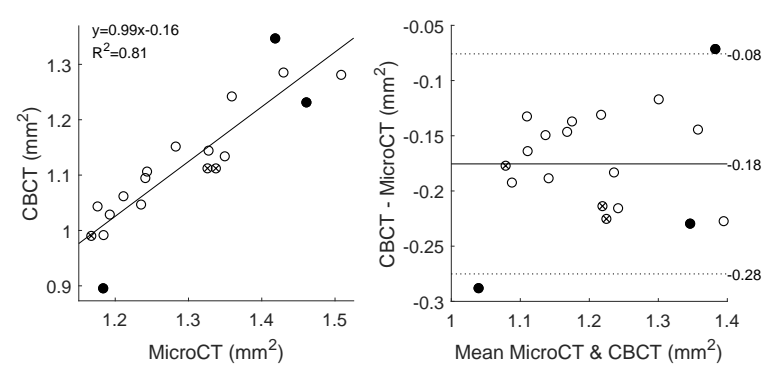

(d) Trabecular number (Tb.N)
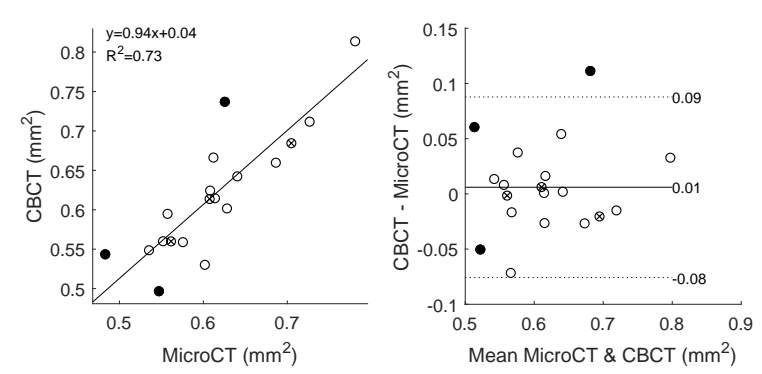

(c) Trabecular separation (Tb.Sp)
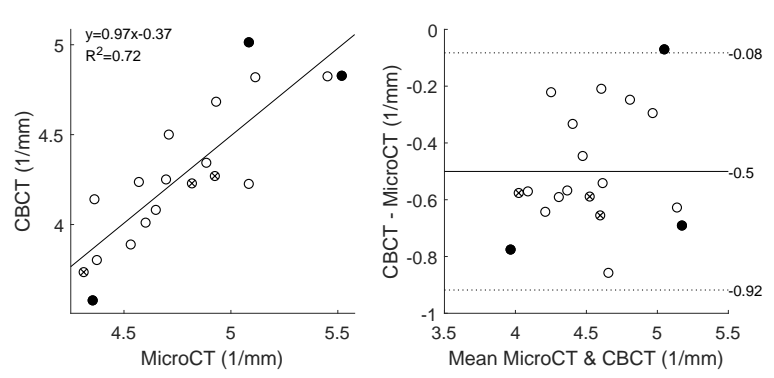

(e) Bone surface density $(B S / T V)$

Figure 2: Regression plots and Bland Altman plots between microCT and RInHouseBeam for bone volume fraction (BV/TV), trabecular thickness (Tb.Th), trabecular separation (Tb.Sp), trabecular number (Tb.N), and bone surface density $(B S / T V)$. Three samples with low trabecular thickness (more than $40 \%$ of the trabeculae in the VOI thinner than $120 \mu \mathrm{m}$ ) are indicated with filled dots. The three samples that contained detailed architectural features (almost parallel trabeculae close to each other, small holes and thin, irregular trabeculae) are indicated with a cross. [2-column fitting image, color] 


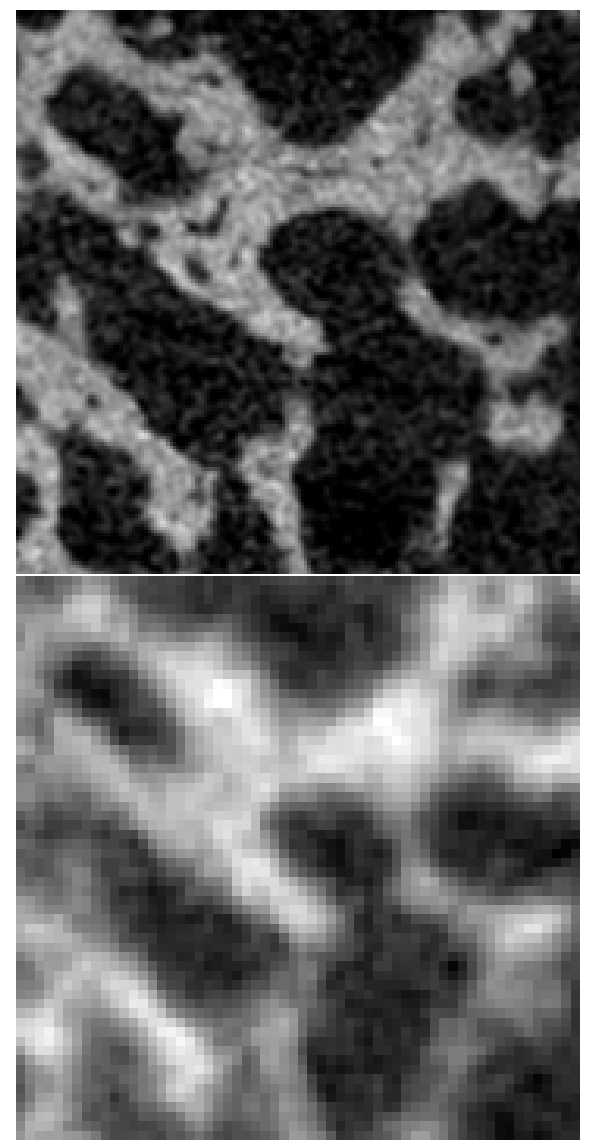

(a) Small holes

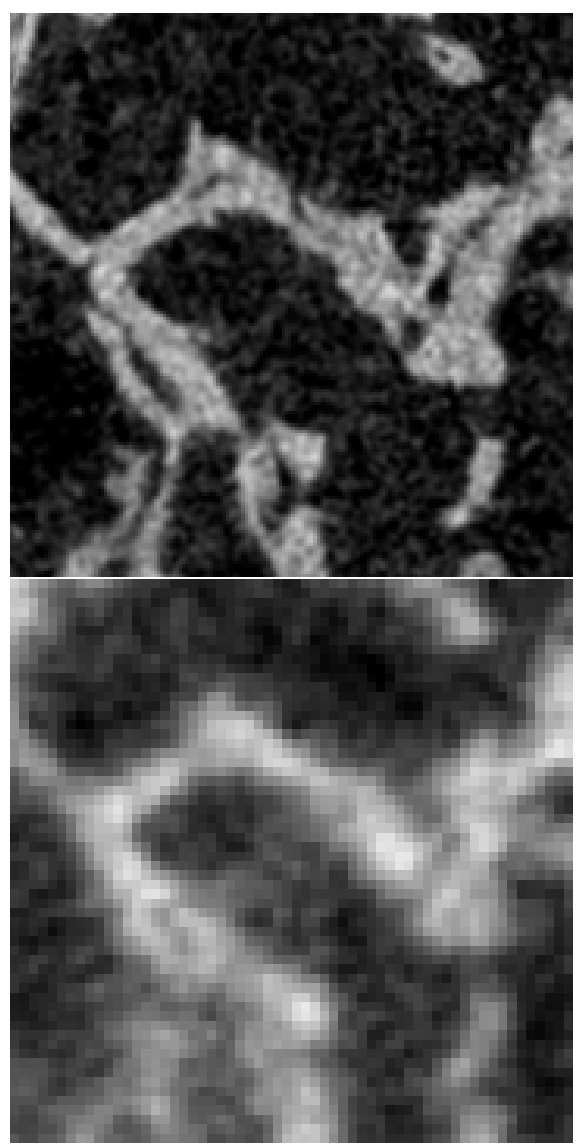

(b) Nearly parallel trabeculae

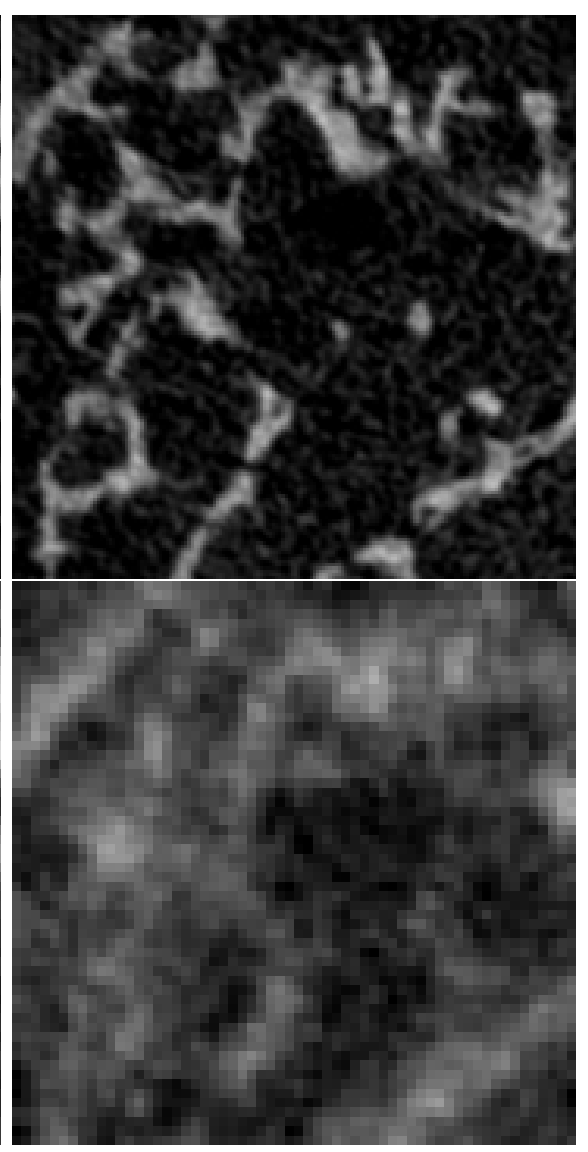

(c) Thin irregular trabeculae

Figure 3: Small bone features such as small holes in the trabeculae (a), nearly parallel trabeculae close to each other (b) and thin, irregular trabecular structures (c) were observed in the microCT-images (top). The resolution of the CBCT-images (bottom) is too low to depict these features, thus causing morphometric differences between CBCT and microCT. [2-column fitting image]

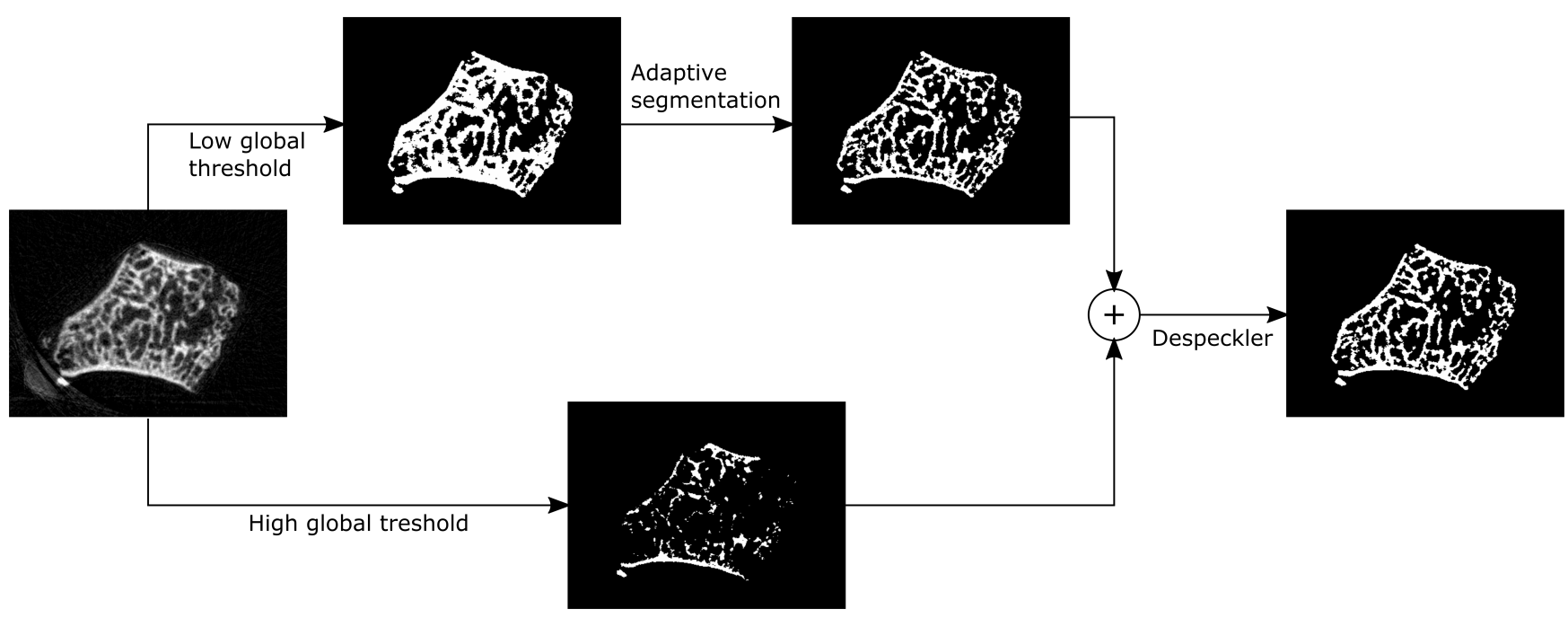

Figure 4: Automatic segmentation technique for the CBCT-images. The main part consists of a low global threshold, followed by an adaptive segmentation in a sphere with a radius of 6 pixels. In parallel a high global segmentation is adapted because the adaptive segmentation can leave thick bone structures undetected. Finally the noise is reduced with a despeckler. [2-column fitting image] 


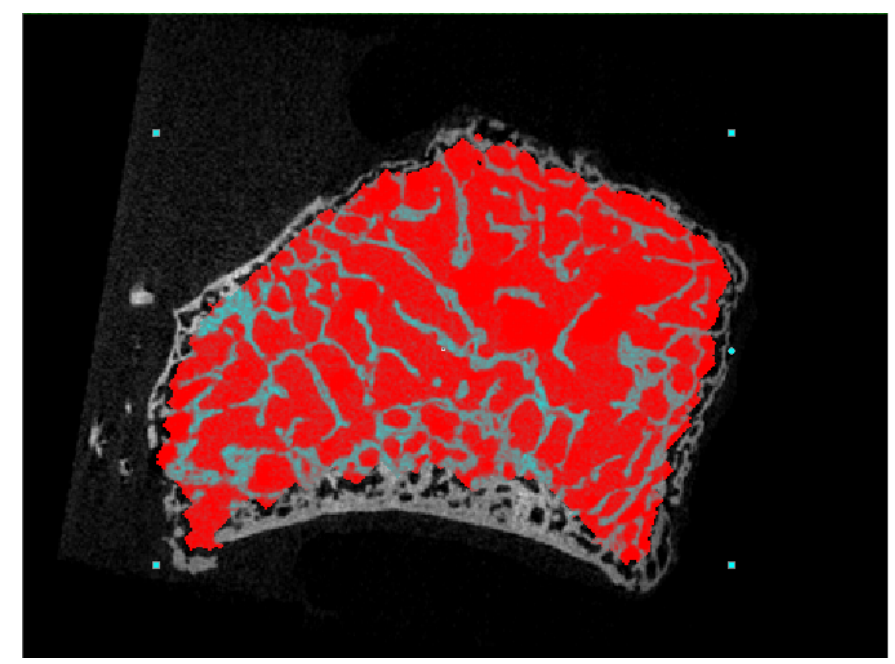

Figure 5: Depicted in red is one section through the volume of interest (VOI) which was used for the microstructural analyses. This VOI is determined automatically from the microCT-images. [1-column fitting image, color] 


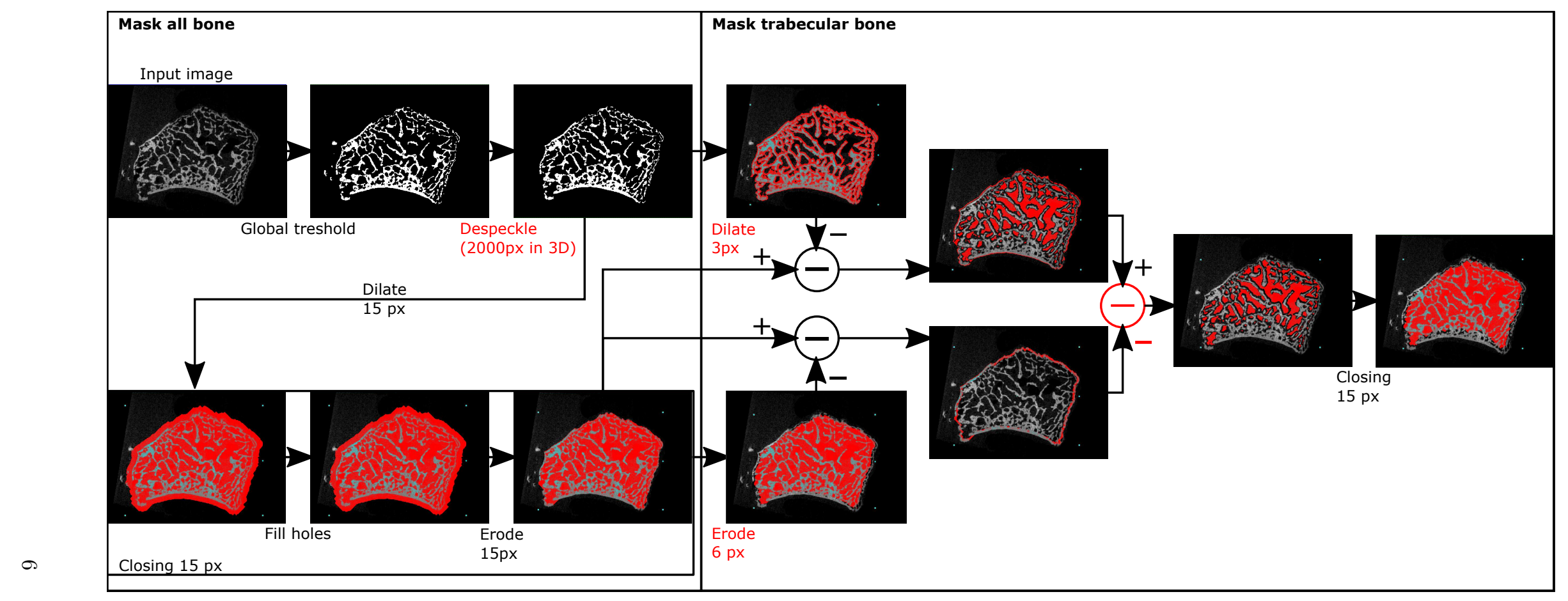

Figure 6: Automatic determination of the volume of interest (VOI). Based on the method by Buie et al [14] several steps were added (marked with red labels) to adapt it for the analyses of CBCT-images. [2-column fitting image, full page, color] 